\title{
Simple Closed-Form Approximation to Ricean Sum Distributions
}

\author{
José A. López-Salcedo, Member, IEEE
}

\begin{abstract}
This letter focuses on the approximation of Ricean sum distributions of $L$ independent random variables. Although no closed-form expression is available for these exact distributions, a simple but accurate closed-form approximation is proposed. The expression is based on the Central Limit Theorem (CLT) for which an appropriate correction term is added. The result is a rather tight fit with the exact distribution for a wide range of input values and Ricean $K$-factors.
\end{abstract}

Index Terms-Approximation methods, Rayleigh distribution, Ricean distribution, sum distribution.

\section{INTRODUCTION}

Ricean statistics are commonly found in wireless communications problems where line-of-sight (LOS) propagation is experienced. This is the case of wireless transmission in the presence of fixed scatterers, where the received signal can be modeled as a fixed component plus zero-mean Gaussian random disturbances. The envelope of the received signal is then Ricean distributed with the following probability density function (PDF) [1],

$$
f_{R}(r)=\frac{r}{\sigma^{2}} e^{-\left(r^{2}+s^{2}\right) / 2 \sigma^{2}} I_{0}\left(\frac{r s}{\sigma^{2}}\right), \quad r \geq 0
$$

where $\sigma^{2}$ is the variance of the zero-mean Gaussian disturbance, $s^{2}$ is the noncentrality parameter and $I_{0}(x)$ is the zeroorder modified Bessel function of the first kind. Two additional parameters are often defined to characterize Ricean statistics. These are the Ricean $K$-factor, $K \doteq s^{2} / 2 \sigma^{2}$, and the average total power $\Omega \doteq s^{2}+\sigma^{2}$.

Contrary to other distributions, the main problem with the Ricean PDF is the presence of the modified Bessel function which makes the mathematical treatment rather cumbersome. This problem aggravates when the sum of multiple Ricean variables has to be faced. This happens for instance when adopting envelope combining strategies for multi-antenna receivers [2] or when extending the correlation period in GNSS receivers [3]. In these circumstances no closed-form expression is available for the resulting distribution and there is no choice but to resort to numerical evaluations or approximate models to avoid Ricean statistics (e.g. using the Nakagami distribution instead).

(Corrected version on November 25, 2009). Manuscript received September 15, 2008; revised November 25, 2008. Current version published February 11, 2009. This work was supported in part by the Spanish/Catalan Government under projects TEC2008-06305 and 2005SGR-00356. The associate editor coordinating the review of this manuscript and approving it for publication was Dr. Shahram Shahbazpanahi.

The author is with Signal Processing for Communications and Navigation (SPCOMNAV), Universitat Autnoma de Barcelona (UAB), 08193 Bellaterra (Barcelona), Spain (e-mail: jose.salcedo@uab.es).
In order to circumvent this limitation, approximations for the sum distribution of Ricean random variables have been proposed in the recent literature. Among these contributions, certainly the one by $\mathrm{Hu}$ and Beaulieu [4] is the one that provides the most accurate performance while still keeping a closed-form expression. This approximation is inspired by the relationship between equal gain combining (EGC) and maximal ratio combining (MRC) in the low SNR regime [5]. The result is a link between Ricean and noncentral chi-square distributions which allows the authors in [4] to propose an approximate Ricean cumulative density function (CDF) based on the generalized Marcum Q-function.

The purpose of this letter is to provide a simpler approximation to the sum distribution of Ricean random variables. Instead of using the generalized Marcum Q-function as in [4], this contribution proposes the adoption of the simpler and widespread Gaussian complementary cumulative distribution. That is, the $Q(x)$ function. The rationale behind the proposed approximation is to exploit the asymptotic similarity between Ricean and Gaussian distributions, according to the Central Limit Theorem (CLT) for the sum of independent and indentically distributed random variables.

The proposed closed-form approximation is then adjusted in order to match the exact distribution. To do so, a correction term is incorporated which is driven by some constant parameters that can be determined via nonlinear least squares optimization. In some sense, the role of this correction term is to optimally summarize in a single expression, the refinements provided by subsequent terms of infinite series approximations to CDFs [6]. This approach of including an optimized correction term is similar to the one adopted in [7]. The difference is that [7] focuses on Small Argument Approximations (SAA) for Rayleigh distributions whereas this contribution focuses on a CLT approximation for Ricean distributions.

Finally, the goodness of the proposed approximation is investigated with numerical evaluations. The results show a tight fit with the exact distribution for a wide range of input values and $L \geq 2$ provided that $K>0.5$.

\section{CDF APPROXIMATION}

Let $Z$ be the sum of $L$ independent and identically distributed Ricean random variables $R_{i}$,

$$
Z \doteq \sum_{i=1}^{L} R_{i}=R_{1}+R_{2}+\ldots+R_{L}
$$


TABLE I

COEFFICIENTS FOR THE PROPOSED APPROXIMATION

\begin{tabular}{c||c|c|c||c|c|c|}
\multicolumn{1}{c||}{} & \multicolumn{3}{c||}{$K=-1.25 \mathrm{~dB}$} & \multicolumn{3}{c|}{$K=1 \mathrm{~dB}$} \\
\cline { 2 - 7 }$L$ & $a_{0}$ & $a_{1}$ & $a_{2}$ & $a_{0}$ & $a_{1}$ & $a_{2}$ \\
\hline 2 & 0.0223 & 0.4841 & 1.3632 & 0.0161 & 0.4564 & 1.3681 \\
3 & 0.0178 & 0.4849 & 1.6980 & 0.0129 & 0.4569 & 1.7104 \\
4 & 0.0153 & 0.4859 & 1.9761 & 0.0111 & 0.4573 & 1.9940 \\
5 & 0.0136 & 0.4865 & 2.2195 & 0.0099 & 0.4577 & 2.2418 \\
6 & 0.0124 & 0.4869 & 2.4386 & 0.0090 & 0.4577 & 2.4647 \\
7 & 0.0114 & 0.4872 & 2.6396 & 0.0083 & 0.4579 & 2.6689 \\
8 & 0.0106 & 0.4874 & 2.8263 & 0.0078 & 0.4582 & 2.8587 \\
9 & 0.0100 & 0.4876 & 3.0014 & 0.0073 & 0.4583 & 3.0367 \\
10 & 0.0095 & 0.4877 & 3.1668 & 0.0069 & 0.4584 & 3.2047
\end{tabular}

\begin{tabular}{c||c|c|c||c|c|c|}
\multicolumn{1}{c||}{} & \multicolumn{3}{c||}{$K=3 \mathrm{~dB}$} & \multicolumn{3}{c|}{$K=5 \mathrm{~dB}$} \\
\cline { 2 - 7 }$L$ & $a_{0}$ & $a_{1}$ & $a_{2}$ & $a_{0}$ & $a_{1}$ & $a_{2}$ \\
\hline 2 & 0.0101 & 0.4203 & 1.3814 & 0.0051 & 0.3753 & 1.4084 \\
3 & 0.0082 & 0.4198 & 1.7310 & 0.0042 & 0.3728 & 1.7638 \\
4 & 0.0071 & 0.4197 & 2.0204 & 0.0037 & 0.3712 & 2.0572 \\
5 & 0.0063 & 0.4190 & 2.2828 & 0.0033 & 0.3717 & 2.3144 \\
6 & 0.0058 & 0.4195 & 2.5001 & 0.0030 & 0.3713 & 2.5454 \\
7 & 0.0053 & 0.4190 & 2.7081 & 0.0028 & 0.3711 & 2.7573 \\
8 & 0.0050 & 0.4188 & 2.9013 & 0.0026 & 0.3696 & 2.9545 \\
9 & 0.0047 & 0.4188 & 3.0823 & 0.0025 & 0.3703 & 3.1388 \\
10 & 0.0044 & 0.4188 & 3.2533 & 0.0023 & 0.3696 & 3.3123
\end{tabular}

The proposed closed-form approximation for the CDF of $Z$ can be expressed as,

$$
F_{L}(t)=1-Q\left(\frac{t-\mu_{R} \sqrt{L}}{\sigma_{R}}\right)-F_{\epsilon}(t)
$$

where $t=z / \sqrt{L}$ is the normalized input argument, $\left\{\mu_{R}, \sigma_{R}^{2}\right\}$ are the mean and variance of each $R_{i}$, and $F_{\epsilon}(t)$ is a correction term. Not including this correction term would result in the Central Limit Theorem (CLT) approximation to the CDF of $Z$. The reason is that the first two terms of (3) are indeed the Gaussian CDF with mean and variance given by the sum of means and variances of each $R_{i}$. Formally, the CLT approximation is asymptotically tight for $L \rightarrow \infty$, but corrections are needed for reduced values of $L$. This correction term is empirically found to be well approximated by a function that resembles the second derivative of a Gaussian pulse. The expression is given by

$$
F_{\epsilon}(t)=a_{0}\left[\left(\frac{t-a_{2}}{a_{1}}\right)^{2}-1\right] e^{-\left(t-a_{2}\right)^{2} / 2 a_{1}^{2}}
$$

where constants $\left\{a_{0}, a_{1}, a_{2}\right\}$ control the amplitude, spread and shift, respectively. The appropriate values for these constants are obtained by nonlinear least squares fitting with the exact CDF, based on the interior-reflective Newton method [8]. This can be done, for instance, by using the lsqcurvefit Matlab function. Regarding the exact CDF, it has been obtained by convolving $L$ Ricean PDFs and then performing numerical integration. Results are shown in Table I for $\Omega=1$ in order to reduce the number of possible test cases with $\{\Omega, K\}$

The goodness of the proposed approximation can be observed in Figures 1-3 for Ricean factors $K=\{-1.25,1,3\}$ $\mathrm{dB}$. These figures correspond to the sum of $L=\{2,4,8,10\}$ independent Ricean random variables and they show the exact

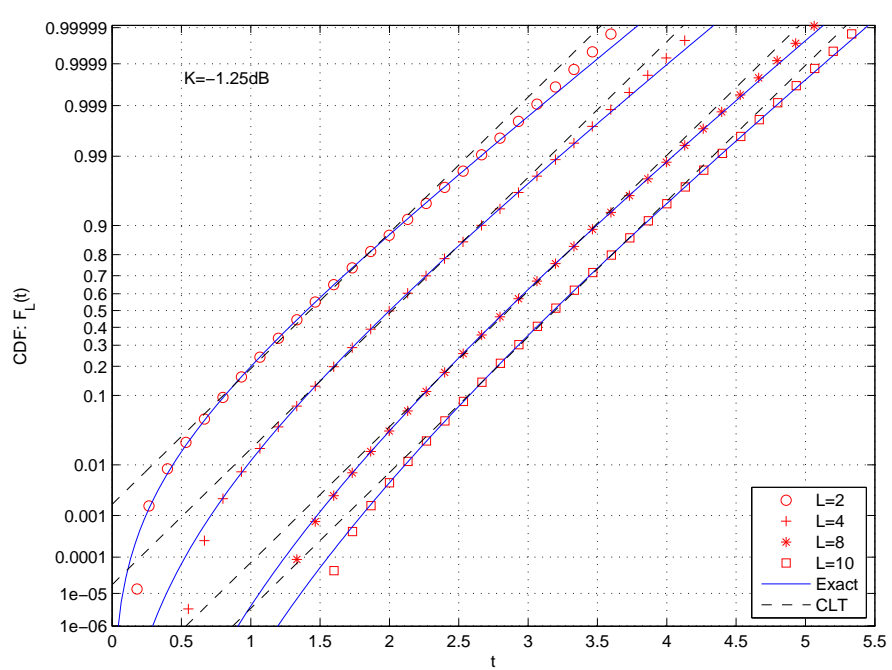

Fig. 1. CDF for the sum of $L=\{2,4,8,10\}$ independent Ricean RV's with $K=-1.25 \mathrm{~dB}$ and $\Omega=1$.

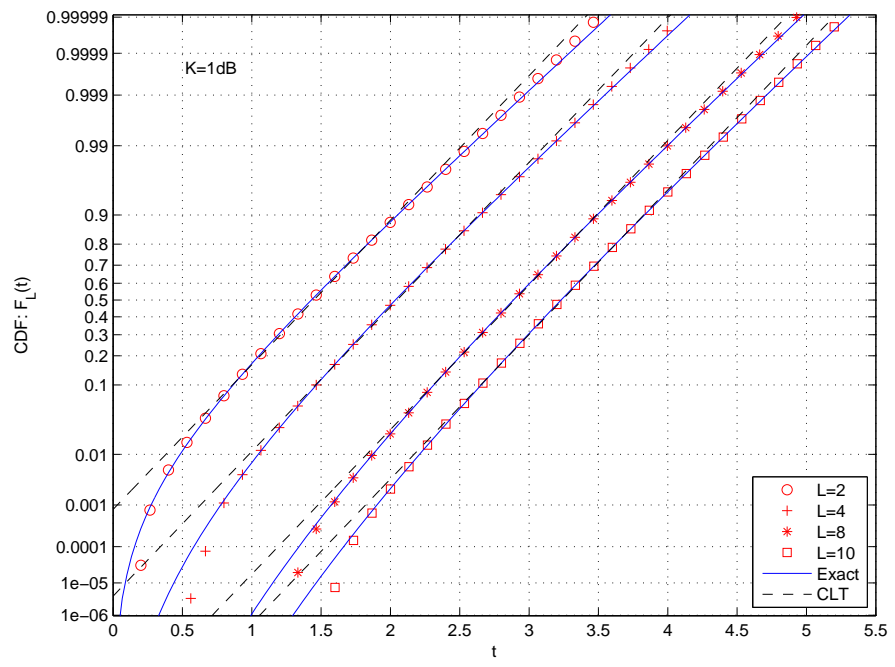

Fig. 2. CDF for the sum of $L=\{2,4,8,10\}$ independent Ricean RV's with $K=1 \mathrm{~dB}$ and $\Omega=1$.

CDF, the CLT approximation and the proposed approximation in (3) with constants $\left\{a_{0}, a_{1}, a_{2}\right\}$ from Table I. Despite of its simplicity, the proposed approximation is found to provide a tight fit for most values of $K$ and $L$. This is especially true for the probability range from $10^{-4}$ to 0.9999 and moderate to large values of $K$. For low values of $K$, however, the proposed approximation starts to deviate from the exact CDF. This effect has also been reported in [4] and it is due to the fact that low values of $K$ (e.g. $K<0.5$ ) correspond to the transition from Ricean to Rayleigh distribution. In that case, Rayleigh approximations or a combination of Rayleigh and Rice approximations should be adopted instead.

\section{PDF APPROXIMATION}

A PDF approximation for the sum of $L$ independent Ricean random variables can be obtained from the $\mathrm{CDF}$ approximation 


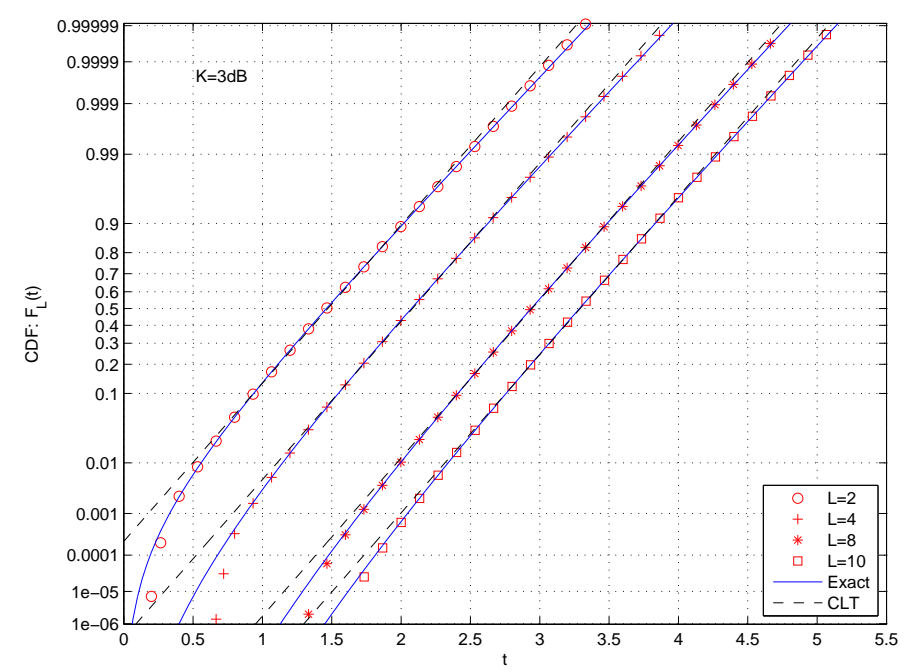

Fig. 3. CDF for the sum of $L=\{2,4,8,10\}$ independent Ricean RV's with $K=3 \mathrm{~dB}$ and $\Omega=1$.

in (3). Let this PDF approximation be denoted by $f_{L}(t)$. Then, by noting that for the exact case $f(t)=\partial F(t) / \partial t$, the proposed PDF approximation turns out to be given by

$$
\begin{aligned}
f_{L}(t)= & \frac{1}{\sqrt{2 \pi}} e^{-\frac{1}{2}\left(\frac{t-\mu_{R} \sqrt{L}}{\sigma_{R}}\right)^{2}}+\frac{a_{0}}{a_{1}}\left[\left(\frac{t-a_{2}}{a_{1}}\right)^{2}-3\right] \\
& \cdot\left(\frac{t-a_{2}}{a_{1}}\right) e^{-\left(t-a_{2}\right)^{2} / 2 a_{1}^{2}} .
\end{aligned}
$$

The goodness of this approximation is shown in Figures 45 for Ricean factors $K=\{1,3\} \mathrm{dB}$ and summations of $L=\{2,4,8,10\}$ Ricean random variables. Again, a tight fit is observed even for low values of $L$ which confirms the validity of the proposed approximation.

\section{CONCLUSIONS}

CDF and PDF closed-form approximations have been proposed for the sum of $L$ independent Ricean variables. The motivation was to provide a simple but accurate approximation to statistical functions for which no closed-form expression is available. The proposed approximation to the Ricean CDF is based on the Gaussian $Q(x)$ function and a correction term which resembles the second derivative of a Gaussian pulse. This correction term is driven by three constants which can be determined via nonlinear least squares optimization. Numerical evaluations confirm the goodness of the proposed approximation that can also be extended to approximate the Ricean PDF through simple differentiation.

\section{REFERENCES}

[1] D. Middleton, "S. O. Rice and the theory of random noise: Some personal recollections," IEEE Trans. Inform. Theory, vol. 34, no. 6, pp. 1367-1373, November 1988.

[2] A. Annamalai, C. Tellambura, and V. K. Bhargava, "Equal-gain diversity receiver performance in wireless channels," IEEE Trans. Commun., vol. 48 , no. 10, pp. 1732-1745, October 2000.

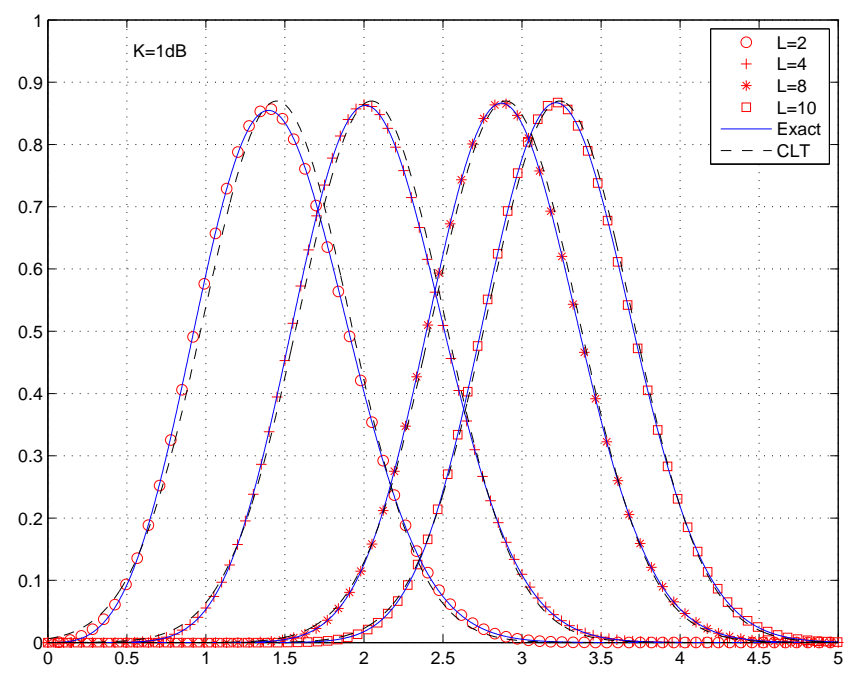

Fig. 4. PDF for the sum of $L=\{2,4,8,10\}$ independent Ricean RV's with $K=1 \mathrm{~dB}$ and $\Omega=1$.

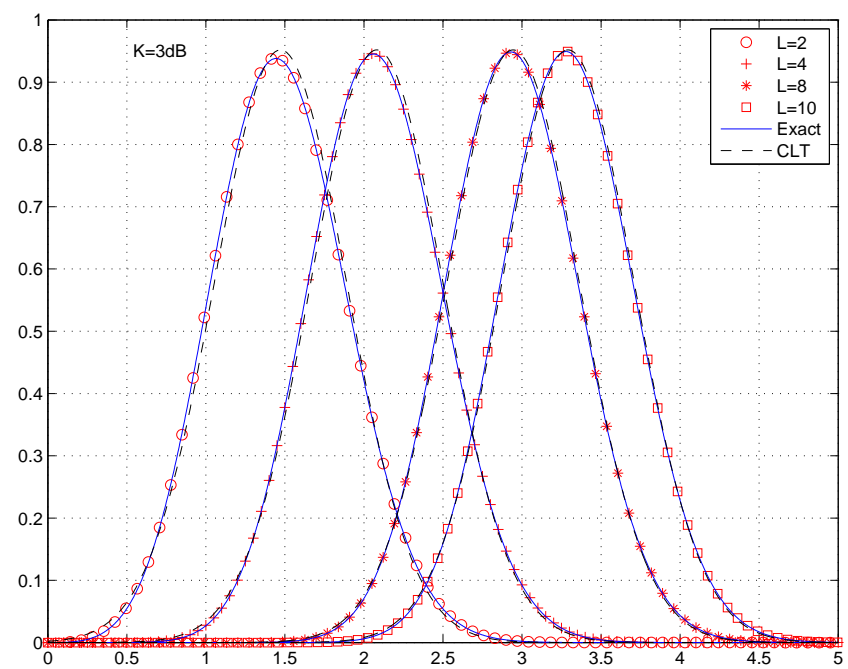

Fig. 5. PDF for the sum of $L=\{2,4,8,10\}$ independent Ricean RV's with $K=3 \mathrm{~dB}$ and $\Omega=1$.

[3] H. Hurskainen, E. Simona, X. Hu, J. Raasakka, and J. Nurmi, "Multiple gate delay tracking structures for GNSS signals and their evaluation with Simulink, SystemC, and VHDL," Intl. Journal of Navigation and Observation, pp. 1-17, 2008.

[4] J. Hu and N. C. Beaulieu, "Accurate closed-form approximations to Ricean sum distributions and densities," IEEE Commun. Lett., vol. 9, no. 9, pp. 133-135, February 2005.

[5] M. Schwartz, W. R. Bennett, and S. Stein, Communication Systems and Techniques. McGraw-Hill, 1966.

[6] N. C. Beaulieu, "An infinite series for the computation of the complementary probability distribution function of a sum of independent random variables and its application to the sum of Rayleigh random variables," IEEE Trans. Commun., vol. 38, no. 9, pp. 1463-1474, September 1990.

[7] J. Hu and N. C. Beaulieu, "Accurate simple closed-form approximations to Rayleigh sum distributions and densities," IEEE Commun. Lett., vol. 9, no. 9, pp. 109-111, February 2005.

[8] T. F. Coleman and Y. Li, "An interior trust region approach for nonlinear minimization subject to bounds," SIAM J. Optimization, vol. 6, pp. 418445, May 1996. 